\title{
A CASE OF PERITONEO-PERICARDIAL DIAPHRAGMATIC HERNIA TREATED SURGICALLY
}

\author{
BY \\ A. EL SHERIF AND S. EL MALLAH \\ From the Cardiac and Thoracic Surgery Units, Manial University Hospital, Cairo University
}

(RECEIVED FOR PUBLICATION MARCH 23, 1956)

Peritoneo-pericardial diaphragmatic hernia is the rarest of all forms of herniation of abdominal organs through the diaphragm.

Only 10 cases have been previously reported. With the exception of three, all were diagnosed after death. Of the three cases diagnosed during life two were diagnosed during operation but the other was diagnosed pre-operatively. The present case to be reported, which constitutes the eleventh in the literature, was diagnosed during operation, and failure to make a pre-operative diagnosis was largely due to unawareness of this rare anomaly (Taillens, 1934). The initial radiological diagnosis was a hydro- or pyo-pneumopericardium.

\section{REVIEW OF THE LITERATURE}

The first case reported in the literature was that of Grenier de Cardenal and Bourderou in 1903, who reported the case of a 58-year-old man taken suddenly ill with abdominal colic and vomiting. Surgical exploration revealed a peritoneo-pericardial hernia containing greater omentum and a loop of transverse colon. The patient died on the day after the operation.

Martland in 1909 (quoted by Wilson, Rumel, and Ross, 1947) reported the case of a 70-yearold man with the clinical picture of right-sided heart failure; at necropsy a defect was found in the diaphragm above the liver through which transverse colon and greater omentum had herniated. There was no hernial sac and the peritoneum of the under surface of the diaphragm was continuous with the parietal pericardium. Keith in 1910 reported three necropsy cases, a 58-year-old man, a 47-year-old man, and a newborn infant. Taillens in 1934 reported a necropsy case of a 2-month-old infant who was suffering from attacks of cyanosis after meals since his birth. O'Brien in 1939 reported a necropsy case of a 64-year-old man, in whom a defect in the anterior leaf of the central tendon was found fused to the floor of the pericardium and through which a part of the liver was protruding. Casey and Hidden in 1944 reported an infant who died four hours after birth. Amongst other anomalies, the central tendon and anterior portion of the diaphragm were lacking, but a pericardio-peritoneal foramen was found without any herniation of the abdominal contents through it.

Wilson, Rumel, and Ross in 1947 reported the first case successfully corrected surgically with recovery of the patient. The patient was a newborn boy who shortly after birth developed cyanosis and later choking spells during feeds, gradually becoming more severe. A chest radio- $\stackrel{\AA}{\varrho}$ graph suggested the possibility of diaphragmatic $\overrightarrow{\overrightarrow{0}}$ hernia. Surgical exploration revealed a pericardio- $\frac{0}{3}$ peritoneal defect through which stomach and small intestine had herniated. The defect was repaired, and nine and a half months later the patient was symptomless and in good health, though herniation had recurred.

The first case pre-operatively diagnosed was reported by Astrup and Ziesler in 1951 in a woman aged 50 , presenting with kyphoscoliosis $\frac{\delta}{2}$ and right-sided heart failure. The radiological appearances resembled a pneumo- $\frac{\text { 이 }}{2}$ pericardium, but detection of gas-filled coils of $\frac{D}{2}$ large intestine overlying the heart near its left border but separated from the lung field by a N narrow brim of soft tissue suggested the correct diagnosis. A barium enema showed herniation ${ }_{N}$ of the transverse colon into the pericardial sace through a defect anteriorly near the sternum.? Complete reduction of the transverse colon ande omentum through a median incision in the epi- $\mathbb{D}$ gastrium was attempted and the defect repaired. There had been no recurrence $14 \frac{1}{2}$ months later.

From this review it can be seen that though this $\frac{0}{\mathbb{D}}$ rare condition may lead to early death it is not $\stackrel{\odot}{\vec{D}}$ incompatible with survival and later it may create $\stackrel{\mathbb{Q}}{2}$ a difficult diagnostic problem in adult and middle life. 


\section{EMBRYOLOGY}

In order to understand the nature of the anomaly the development of the diaphragm should be briefly discussed.

The diaphragm is an organ which develops from several origins, and acts as a partition between the serous cavities of the body.

The intra-embryonic part of the coelom, the precursor of the peritoneal, pleural, and pericardial cavities, appears at first as a series of cleftlike spaces in the margin of the embryonic mesoderm. These spaces fuse together to form an inverted U-shaped cavity which divides the peripheral part of the embryonic mesoderm into parietal or somatic and a visceral or splanchnic area. The bend of the inverted U-shaped cavity lies in the margin of the cephalic part of the embryo, and is the precursor of the pericardial cavity. As the head fold forms, the mesoderm, which is found peripheral to the part of the coelom to be the pericardial cavity, becomes thickened and is called the septum transversum and comes to lie in the cephalic boundary of the umbilical orifice. The septum transversum is therefore a mass of mesodermal origin which intervenes between the pericardium and the ventral portion of the abdominal cavity. From it is derived the caudal (lower) part of the pericardium, the ventral part of the central tendon, lateral parts of the diaphragm, the falciform ligament, the coronary ligaments of the liver, and the mesodermal tissue of the liver. The rest of the diaphragm is developed from the dorsal mesentery, the fourth cervical muscle plate and the pleuroperitoneal folds. It can be surmised that if the septum transversum does not develop there will be a gap in that part of the diaphragm formed by it together with the pericardium and the falciform and coronary ligaments, which form the peritoneal undercover of the diaphragm, and hence there will be a direct communication between the pericardial and peritoneal cavities in this region. This is exactly the condition which was found in the case to be described.

\section{CASE Report}

A schoolgirl aged 18 years was admitted to hospital on April 13, 1952, whose principal complaint was cough and inability to lie on the left side, as such a position caused her uneasiness in breathing. The illness started five months before admission to hospital after a severe choking sensation and a fainting spell from hastily drinking a glass of water. She recovered consciousness in a few minutes, but shortly afterwards developed an evening rise of temperature and a dry cough more pronounced by night, arousing her from sleep and associated with severe suffocation and a sour sensation in the mouth. She learned not to have any dinner, as this used to provoke the nocturnal distress. Distress was also experienced if she lay down shortly after meals. Slight expectoration, at first inoffensive but later offensive, was complained of in the last six weeks before admission.

Examination revealed a normally built young woman, apparently healthy, who had a low-grade fever and a hypochromic anaemia $(50 \%$ haemoglobin level). The total white blood count was normal with $66 \%$ polymorphs. The striking feature was loud gurgling and splashing sounds over the lower left chest unrelated to respiratory movements or cardiac action that, together with the radiological findings in the chest, suggested to one of us the possibility of a stomach inside the chest. But repeated observation afterwards revealed the variability in the intensity of these adventitious sounds.

The plain radiograph of the chest was originally diagnosed as a case of hydro- or pyo-pneumopericardium (Figs. 1 and 2), an explanation which was thought to be unlikely because of the absence of any symptoms or signs of cardiac embarrassment and because of the absence of those systemic disturbances commonly present with inflammation of the pericardial sac. The case was then diagnosed as a displaced heart rather than as a distended pericardium, but in our present knowledge of the real diagnosis it can be seen that on the left side the wall of the sac as it goes towards the diaphragm passes outwards rather than inwards, a point that may be of help in arriving at a true diagnosis in other cases. No airfilled area along the right heart contour and no gasfilled coils of intestine overlying the heart or near the left heart contour could be detected as in the case diagnosed pre-operatively by Astrup and Ziesler (1951). When a barium meal was given the fundus of the stomach was shown to be herniated through the diaphragm, but another air-containing viscus was evident (Fig. 3), which on a later study with a barium enema was found to be the colon (Fig. 4). The stomach was seen partly below and partly above the diaphragm, and the duodenum was visualized below the diaphragm. The oesophagus was found running its normal length and the cardiac orifice was below the diaphragm. The colon was shown to enter through the diaphragm anterior to the stomach, and the stomach after its ascent was bent on itself as it passed down again to below the diaphragm. With this information the plain lateral radiograph was then considered to represent a hiatal type of diaphragmatic hernia, the two fluid levels representing the two portions of the stomach (Fig. 5).

The patient was referred to a chest surgeon for operative correction of what was now presumed to be a large hiatal hernia.

OPERATION.- Under endotracheal gas and oxygen supplemented by intraval and tubocurarine the left side of the chest was opened through the bed of the seventh rib. 
FIG. 1.-Postero-anterior view showing a greatly distended pericardial cavity with a fluid level.

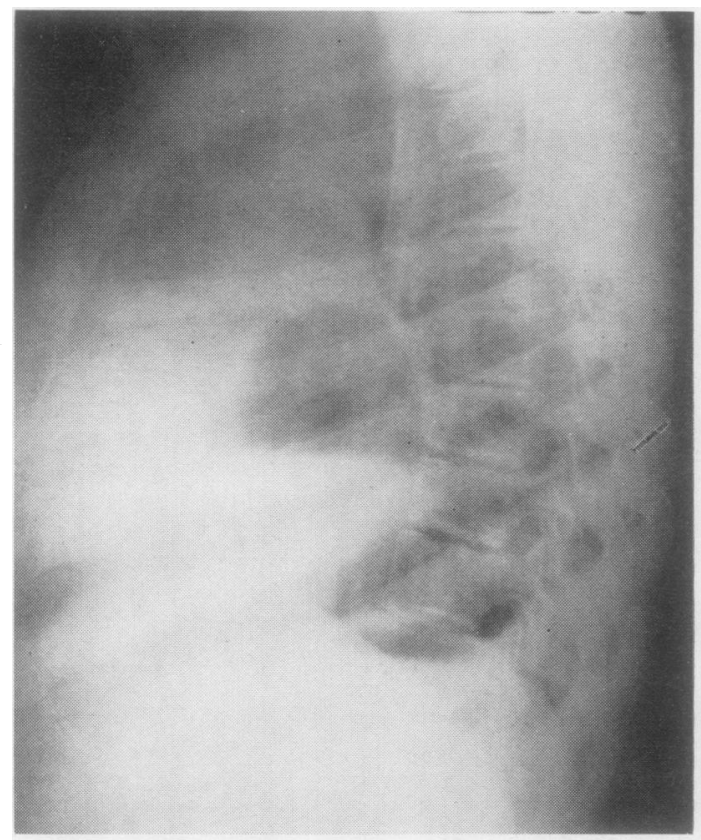

FIG. 2.-Lateral view showing the distended pericardial sac with a fluid level behind the heart shadow.

The lower lobe of the lung was found adherent by thin filamentous adhesions to the chest wall and diaphragm. Adhesions were divided and the lung retracted upwards. After division of the ligamentum latum pulmonis the oesophagus was exposed and followed down to the hiatus, where no abnormality could be detected. At that moment it was noticed that the pericardial sac was distended and peristaltic move-

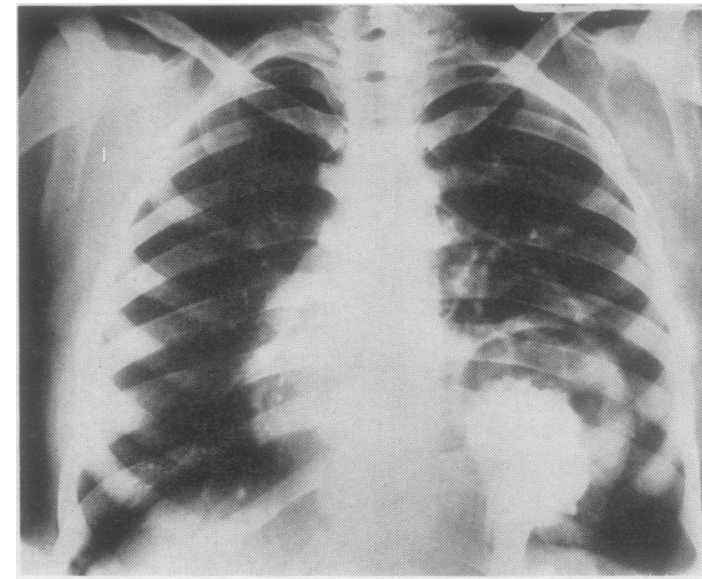

FIG. 3.-Barium outlining the stomach and above it the colon with its characteristic haustrations.

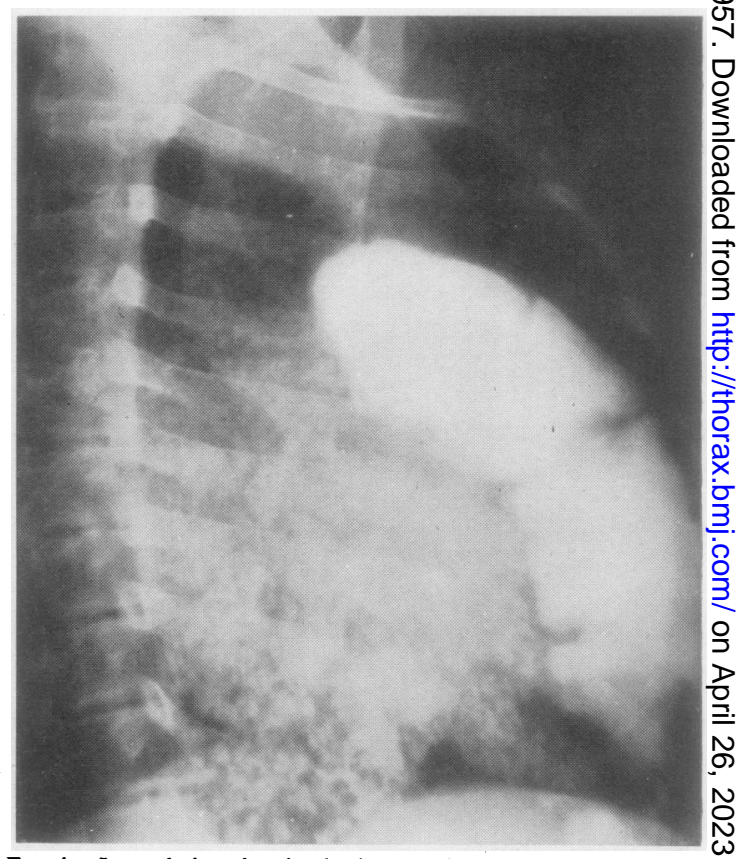
Fig. 4.-Lateral view showing barium outlining the colon high up ing
the chest.

ments were visible through the pericardium. The pericardium was opened by a long incision anterior and parallel to the phrenic nerve, when the stomach, trans- $-\frac{\overrightarrow{0}}{\mathrm{O}}$ verse colon, omentum, small intestine, and part of $\stackrel{?}{?}$ the liver were seen next to the heart uncovered by $a \stackrel{\mathbb{Q}}{\varrho}$ sac. These viscera were reduced, reduction beingO helped by lowering the lower end of the operating table, and then a big defect in the diaphragm com- -0 
municating directly with the pericardial cavity and the general peritoneal cavity was seen. The defect was oval, affecting the anterior part of the central tendon and measuring about 3 by 6 in. The rent was sutured by strong silk interrupted sutures. It was securely closed, but no overlapping could be done. The pericardium was closed, leaving a part at the lower end to drain into the chest, which was closed after placing an underwater seal (Fig. 6).

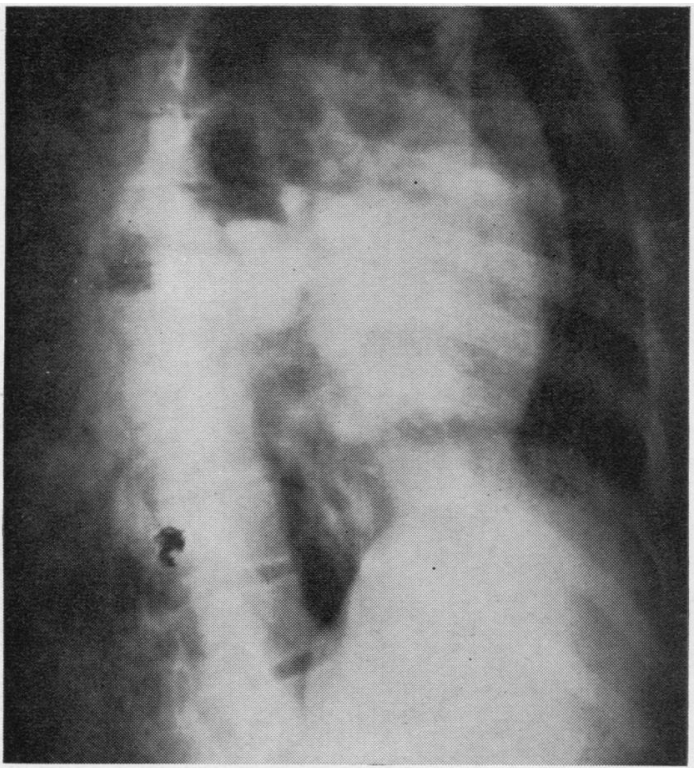

FIG. 5.-Oblique view after barium meal showing multiple fluid levels.

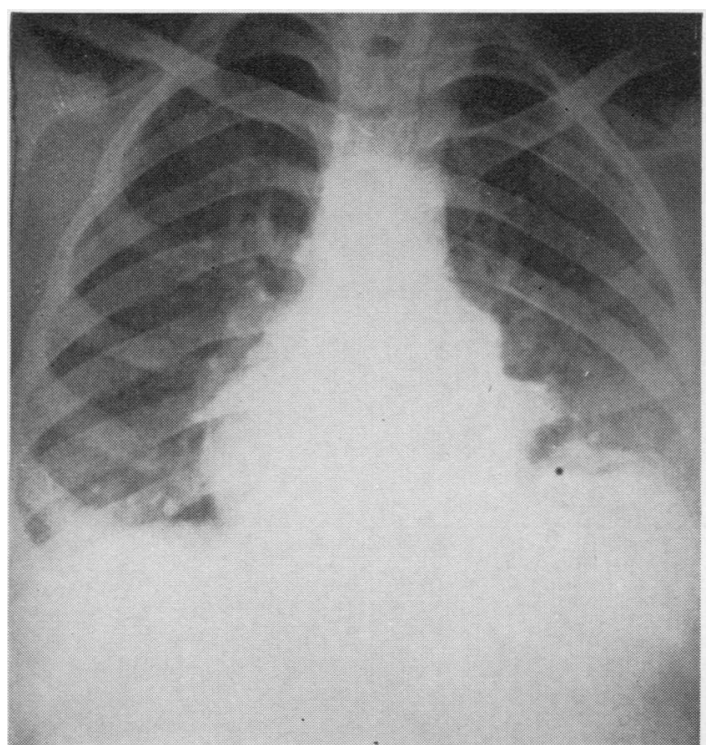

Fig. 6.-Antero-posterior radiograph after operation.

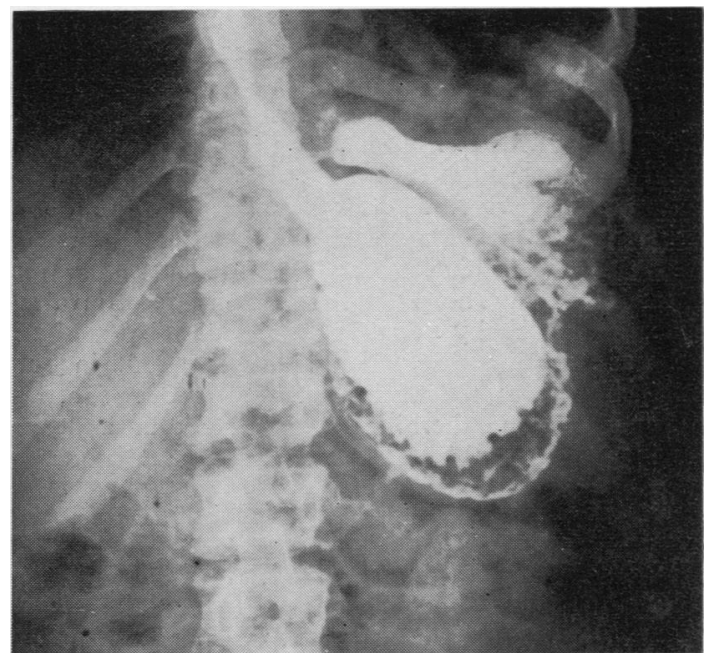

Fig. 7.-Barium after operation showing the twisted stomach and the high pylorus reaching almost to the level of the cardia and the stomach below the diaphragm.

Post-operative Course and Second Operation.The underwater seal was removed in 24 hours. Apart from a low-grade fever that persisted for some weeks, the immediate post-operative course was smooth, but later the patient started to complain of colicky pains, discomfort, and upper abdominal swelling after meals. Examination after meals revealed a big swelling with a definite lower margin, occupying the epigastric and left hypochondriac areas and merging gradually underneath the left costal margin. A barium meal showed that the stomach was now all in the abdomen but kinked on itself with the duodenum at a higher level than normal and the pylorus at almost the same transverse level as the cardia (Fig. 7). In other words, a condition of volvulus of the stomach on its transverse axis was present. The colicky pains and the swelling resulted from the efforts of the proximal stomach pouch to push its contents into the distal one. This condition seems to have resulted from (1) the high position of the duodenum, opposite the tenth dorsal vertebra, due to the continuous suction of the abdominal contents up into the chest by the negative intrathoracic pressure; (2) pushing the stomach down during operation resulted in the creation of two pouches, one distal and anterior and the other proximal and posterior, with a kink in between the two. This state of affairs was proved at the second operation.

As the patient continued to complain of the colicky pains and the swelling after meals, a gastrojejunostomy was decided upon between the jejunum and the proximal gastric pouch. The abdomen was opened through a median supra-umbicilal incision. The rent in the diaphragm which had been sutured in the first operation was found to be giving way. The stomach 
was found to be twisted on its transverse axis, with a bigger upper and a smaller lower compartment. A posterior " no-loop" gastrojejunostomy was performed on the upper pouch. The diaphragmatic rent was repaired again with overlapping of the edges, which was found easier through the abdominal route. Appendicectomy was performed as well, because the patient, during her hospital stay after the first operation, had had an attack of acute appendicitis which was treated conservatively. The patient had a smooth convalescence. The colicky pains and the swelling vanished. She was discharged on November 2, 1953, in full health, having gained weight. One year later she was examined and the herniation did not recur and she was in perfect health.

\section{SUMMARY}

A case of peritoneo-pericardial diaphragmatic hernia successfully corrected surgically is reported. It constitutes the eleventh case in the literature of this rare anomaly, the fourth diagnosed during life, and the third successfully treated.
A review of the literature and a short account on the development of the diaphragm are given.

Awareness of the existence of this rare anomaly should ensure that in future such cases are diagnosed pre-operatively. A distended pericardial sac with no evidence of pericarditis but post-prandial cardiac or respiratory distress should make such a condition a diagnostic possibility. Detection of gas-filled coils separated from the lung tissue by a thin rim of soft tissue that merges through the diaphragm outwards rather than medialwards seems to be sufficiently diagnostic.

\section{REFERENCES}

Astrup, E. C., and Ziesler, E. (1951). Acta med. scand., 141, 153. Casey, A. E., and Hidden, E. H. (1944). Arch. Path. (Chicago), 38 370.

Grenier de Cardenal, H., and Bourderou (1903). J. Méd., Bordéaux, 33, 222.

Keith, A. (1910). Brit. med. J., 2, 1297.

O'Brien, H. D. (1939). J. Anat. (Lond.), 74, 131.

Taillens, J. (1934). Bull. Soc. Pédiat, Paris, $39,558$.

Wilson, . Amer. J. Roentgenol., 57, 42. 